\title{
Real-time oncological guidance using diffuse reflectance spectroscopy in electrosurgery: the effect of coagulation on tissue discrimination
}

Maartje W. Adank

Julie C. Fleischer

Jenny Dankelman

Benno H. W. Hendriks 


\title{
Real-time oncological guidance using diffuse reflectance spectroscopy in electrosurgery: the effect of coagulation on tissue discrimination
}

\author{
Maartje W. Adank, ${ }^{a}$ Julie C. Fleischer, ${ }^{a}$ Jenny Dankelman, ${ }^{a}$ and Benno H. W. Hendriks ${ }^{a, b, *}$ \\ ${ }^{a}$ Delft University of Technology, Biomechanical Engineering Department, Delft, The Netherlands \\ ${ }^{\mathrm{b}}$ Philips Research, In-Body Systems Department, Eindhoven, The Netherlands
}

\begin{abstract}
In breast surgery, a lack of knowledge about what is below the tissue surface may lead to positive tumor margins and iatrogenic damage. Diffuse reflectance spectroscopy (DRS) is a spectroscopic technique that can distinguish between healthy and tumor tissue making it a suitable technology for intraoperative guidance. However, because tumor surgeries are often performed with an electrosurgical knife, the effect of a coagulated tissue layer on DRS measurements must be taken into account. It is evaluated whether real-time DRS measurements obtained with a photonic electrosurgical knife could provide useful information of tissue properties also when tissue is coagulated and cut. The size of the coagulated area is determined and the effect of its presence on DR spectra is studied using ex vivo porcine adipose and muscle tissue. A coagulated tissue layer with a depth of 0.1 to $0.4 \mathrm{~mm}$ is observed after coagulating muscle with an electrosurgical knife. The results show that the effect of coagulating adipose tissue is negligible. Using the fat/water ratio's calculated from the measured spectra of the photonic electrosurgical knife, it was possible to determine the distance from the instrument tip to a tissue transition during cutting. In conclusion, the photonic electrosurgical knife can determine tissue properties of coagulated and cut tissue and has, therefore, the potential to provide real-time feedback about the presence of breast tumor margins during cutting, helping surgeons to establish negative margins and improve patient outcome. () The Authors. Published by SPIE under a Creative Commons Attribution 3.0 Unported License. Distribution or reproduction of this work in whole or in part requires full attribution of the original publication, including its DOI. [DOI: 10.1117/1 JBO.23.11.115004]
\end{abstract}

Keywords: diffuse reflectance spectroscopy; coagulation; intraoperative guidance; electrosurgical knife.

Paper 180281RR received Jun. 3, 2018; accepted for publication Oct. 15, 2018; published online Nov. 16, 2018.

\section{Introduction}

Breast cancer is expected to affect one in eight women during their lifetime, ${ }^{1}$ making it a major global health threat. Breast conserving surgery (BCS) is the preferred choice of surgical treatment. $^{2-4}$ The main challenge in BCS is to ensure minimal healthy tissue removal while extracting the tissue lump with negative margins. A negative margin means that the excised tumor is completely surrounded by a small layer of healthy tissue, leaving no tumor cells behind in the body. ${ }^{2}$ Positive margins create higher chances of local recurrence, a decreased likelihood of overall survival, and require additional treatments such as reexcision surgery, extra radiation therapy, and chemotherapy. ${ }^{5-9}$ Tartter et al. ${ }^{10}$ found positive margin prevalence rates ranging from $31 \%$ to $46 \%$ for ductal carcinoma in situ (DCIS) alone and $11 \%$ to $46 \%$ for invasive breast cancers and DCIS combined. Kobbermann et al. ${ }^{11}$ found that $31.9 \%$ of the patients who had a partial mastectomy required repeat surgery. These rates indicate that positive margins in breast cancer surgery are a wide-spread problem that leads to high costs and negative patient outcomes.

Surgeons do not have a direct view of the tissue below the surface and have to rely on anatomical knowledge and imaging technology to estimate the position of tumors and critical structures. Real-time information about the location of the tumor margins provided during surgery can reduce the number of

*Address all correspondence to: Benno H. W. Hendriks, E-mail: benno .hendriks@philips.com positive margins. Methods that are currently in use to locate margins during surgery include palpation, ultrasound (US), guidewires, iodine seed localization, radio occult lesion localization (ROLL), and preoperative images. ${ }^{12-14}$ Intraoperative margin assessment is currently done using either touch prep cytology or frozen section analysis (cryosection), ${ }^{15,16}$ but it is not standard practice and only used in a few hospitals. ${ }^{17}$ In touch prep cytology (also called imprint cytology), a glass slide is touched to the surface of an excised specimen and microscopically assessed by a cytologist. Although this method covers the complete surface of a specimen, underlying cells are not taken into account. In frozen section analysis, tissue slices fixed in compound are studied microscopically. Both methods are time-consuming as analysis time ranges from 20 to $40 \mathrm{~min}$ and only provide information on part of the surgical margin. ${ }^{18-20}$ Except for Moh's surgery for skin lesions, these methods are not widely used. The MarginProbe (Dune Medical) allows faster intraoperative assessment of the margins on the lump, but it is not commonly applied. ${ }^{21}$ The iKnife (under development) analyzes the smoke produced by electrosurgical cutting to establish whether or not tumor tissue was cut. ${ }^{22}$ Except for intraoperative US and ROLL, none of these techniques provide real-time in vivo information about the tumor margin near the instrument tip.

Diffuse reflectance spectroscopy (DRS) is an optical spectroscopy technique that has been shown to accurately discriminate healthy tissue from tumor tissue. ${ }^{23-28}$ In a study that compared five types of breast tissue (adipose, glandular, fibroadenoma, invasive carcinoma, and DCIS), Nachabé et al. ${ }^{25}$ found 
sensitivities ranging from $83 \%$ to $98 \%$ and specificities between $95 \%$ and $99 \%$. Integration of this technique into a surgical instrument tip may, for instance, enable real-time sensing of tumor margins, helping surgeons to establish clear margins and avoid iatrogenic injury in a more direct and intuitive way than for instance US or ROLL. De Boer et al. ${ }^{29}$ and de Boer et al..$^{30}$ have shown that tumor borders in lumpectomy specimens can be identified using the fat/water-ratio extracted from the near-infrared (NIR) spectrum of DRS measurements. In their study, a needle-like probe was used to obtain DR spectra. However, in most cancer surgeries, an electrosurgical instrument is used to cut tissue, resulting in an area of coagulated tissue surrounding the electrode. ${ }^{31,32}$ Coagulation of tissue increases scattering multiple times and reduces photon penetration depth compared to normal tissue, significantly altering DR spectra and requiring us to treat coagulated tissue as a distinct layer. ${ }^{33-37}$

In this paper, the size of the tissue area affected by electrosurgery and the effect of coagulation on DR spectra are studied using layered porcine adipose and muscle tissue to simulate breast cancer surgery. An electrosurgical knife with an embedded real-time DRS sensing system is presented for the first time as well as its abilities to provide information on the distance between the instrument tip and the tissue layer of interest. The aim of this study is to establish the capabilities of DRS to sense through the coagulated layer, providing insight into the usability of this technique in surgery with electrosurgical instruments.

\section{Method}

\subsection{Specimen}

Three models were created to perform the experiments [Figs. 1(d)-1(f)]. First, to test the effect of coagulation on muscle and adipose tissue, measurements obtained during cutting were performed on swine bacon tissue because it consists of alternating layers of adipose and muscle tissue.
The second model was created to test the DRS signal in muscle and adipose tissue. In this setup, normal breast tissue is simulated using porcine adipose tissue, and tumor tissue is simulated by porcine muscle tissue. To simulate tumor tissue, 2-cm layers of porcine muscle tissue (LifeTech, Eindhoven) were used; this tissue is rich in proteins and blood, similar to tumor tissue. Normal tissue was simulated by the use of 2-cm layers of porcine adipose tissue (LifeTech, Eindhoven). This model was also used for the fourth experiment (see Sec. 2.5), where the electrosurgical knife is advanced through fat and muscle layers from a different side.

In the third model, the aim was to simulate at the transition from normal breast tissue to tumor tissue. The same 2-cm layers of porcine muscle tissue (LifeTech, Eindhoven) were used to simulate tumor tissue. To simulate the transition from breast tissue to tumor tissue, 0.4-mm thin layers of sowbelly tissue were used and obtained from the abattoir (Butcher "Lelieveld," Eindhoven). The sowbelly tissue was the fattiest tissue available that could be sliced in thin layers.

All specimens were preserved in a frozen state. The tissue was thawed for at least $2 \mathrm{~h}$ prior to the experiments.

\subsection{Electrosurgical Treatment of Tissue}

Two types of usage of the electrosurgical knife were studied: cutting [Fig. 1(b), left] and coagulation [Fig. 1(b), right]. A cutting current quickly heats up individual cells, which first leads to boiling and then causes cell rupture. This setting is used to cut the tissue quickly with minimized charring. ${ }^{38,39}$ For coagulation, the "fulguration coagulation" setting was applied on the electrosurgical force generator and the knife was held slightly above the tissue. The current was applied to the tissue in an on-off mode. The off-time allows the cells to cool down when an interruption is present, which causes more extensive charring and neighboring tissue damage. Clinically, it is mainly used to stop bleeding. ${ }^{38,39}$

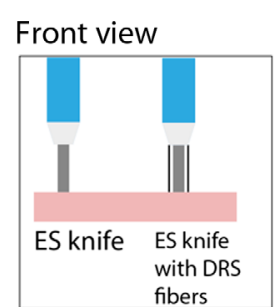

(a)

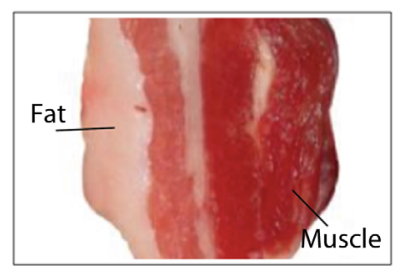

(d)

(b)
Close up schematic side view
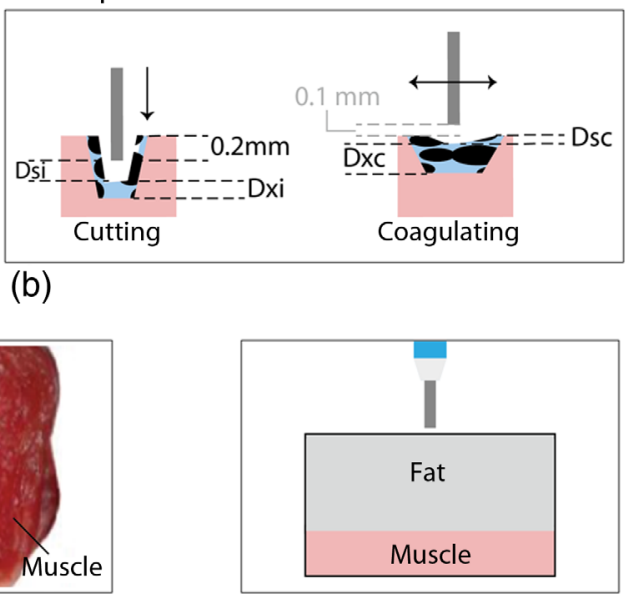

(e)

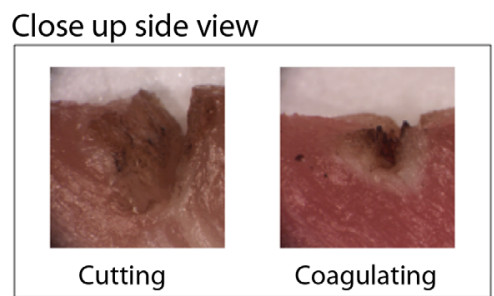

(c)

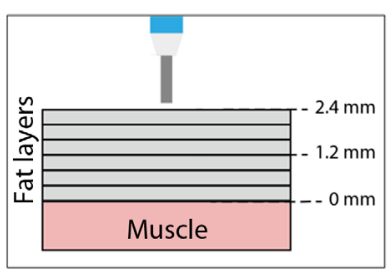

(f)

Fig. 1 (a) Schematic drawing of the electrosurgical knife without and with optical fibers, (b) schematic drawings of the tissue change during incision by cutting Dsi and coagulation Dsc, with pink representing normal tissue, and blue/black representing the tissue that has been coagulated, the depth of the coagulation zone for incision Dxi and for coagulation Dxc, (c) photographs of the tissue change due to cutting and coagulation, (d) model 1: top view of porcine muscle tissue, (e) model 2: side view schematic drawing of 0.4-mm fat layers on muscle tissue, and (f) model 3: side view schematic drawing of porcine fat and muscle tissue. 
In the experiment, the change in optical properties of ex vivo porcine tissue due to cutting and coagulating was investigated. The focus was on the formation of a coagulation zone shown in Figs. 1(b) (right) and 1(c) (right). Both cutting and coagulation of the tissue were applied with the blade perpendicular to the tissue surface as shown in Fig. 1(a). Cutting and coagulation were done using a linear stage actuator (Newport, Irvine, USA) to control the movement speed and distance with respect to the tissue. Cutting was performed with the electrode tip placed $0.2 \mathrm{~mm}$ into the tissue. Coagulation was done with the electrode tip placed $0.1 \mathrm{~mm}$ above the tissue. To prevent the contamination of the knife from influencing the results, the electrode blade was cleaned with alcohol and a soft cloth when necessary. The equipment used consisted of a Valleylab ForceX generator (Medtronic, Minnesota, USA) combined with an Erbe disposable pencil and a 4-mm blade electrode (Erbe, Tübingen, Germany). Based on observations in clinical practice and literature, power settings ranging from 40 to $80 \mathrm{~W}$ with $10-\mathrm{W}$ increments were used. ${ }^{40-42}$ Each power setting/tissue type/electrosurgical combination was applied 10 times.

\subsection{Coagulation Depth Estimation}

The treated tissue was frozen for at least $2 \mathrm{~h}$, allowing us to cut solid slices through the coagulated layer. Microscopic images of these slices were taken with a ruler included in the image. Distances were calculated with Image-J (National Health Institute, USA). The measurements taken were: the depth of the coagulation zone under a cut (Dxi), the incision depth after cutting (Dsi), the depth of the coagulation zone after coagulating (Dxc), and the incision depth after coagulating (Dsc). These variables are schematically shown in Fig. 1(b). Coagulation zone depth was measured from the top to the bottom of coagulated tissue in at least three different locations in the microscopic images.

\subsection{Spectroscopic System}

Diffuse reflectance (DR) spectra ranging from 400 to $1600 \mathrm{~nm}$ were obtained using an optical system consisting of: a broadband halogen light source (Avantes, Apeldoorn, The Netherlands), a spectrometer designated for light collection in the 400- to 1000-nm range (Mayapro 2000, Ocean Optics, Dunedin, USA), and a spectrometer that collects light between 900 and $1650 \mathrm{~nm}$ (NIRQuest 512, Ocean Optics, Dunedin, USA). DR spectra were recorded at 1-nm wavelength intervals. The light source is controlled with an internal shutter. The system was controlled with LabView (National Instruments, Austin, Texas, USA) using a custom designed Philips PhotonicNeedle software (PNSas), a software interface developed specifically for this purpose.

Two devices were used in this study. The first is a flat-tipped optical probe with a fiber-to-fiber distance (FD) of $6.2 \mathrm{~mm}$. The second is the photonic electrosurgical knife (Philips Research, Eindhoven) with an FD of $3 \mathrm{~mm}$. All fibers had a core and cladding diameter of 200 and $220 \mu \mathrm{m}$, respectively. Calibration was done with the use of a white reflectance standard (Spectralon), as described in Ref. 25. The integration time, i.e., the time that the spectrometers collected photons, is set on $1 \mathrm{~s}$ for the $6.2-\mathrm{mm}$ probe and $0.5 \mathrm{~s}$ for the $3-\mathrm{mm}$ probe for both the visible (VIS) and the NIR region. One of the fibers of the optical probe was coupled to the white light source and the other via a custommade fiber splitter to the VIS and NIS spectrometer.
The probe and the photonic electrosurgical knife were placed perpendicular to the tissue, in contact with the tissue surface without exerting pressure. This was done by letting the probe or photonic electrosurgical knife rest on the tissue while making sure the tissue was not dented.

\subsection{Spectroscopic Measurements}

To investigate the influence of the use of an electrosurgical knife when approaching a tumor in the breast during BCS, four experiments were conducted. In the first experiment, model 2 was used where a layer of 2-cm adipose tissue laid on a layer of $2-\mathrm{cm}$ muscle tissue. The spectra were obtained from thick $(\sim 1 \mathrm{~cm})$ slices of muscle and adipose tissue before and after coagulation at power settings ranging from 50 to $80 \mathrm{~W}$ at $10-\mathrm{W}$ increments. Measurements were done with the flattipped probe.

The second experiment included measurements of DR spectra during the transition from pure adipose tissue to pure muscle tissue without the use of electrosurgery. Five sowbelly tissue layers of $0.4 \mathrm{~mm}$ were stacked on top of a $2-\mathrm{cm}$ muscle layer to simulate the transition between the tissues, shown as model 3 in Fig. 1. DR spectra were acquired, one layer of sowbelly was removed, and DR spectra acquired again. This process was repeated until the probe was placed directly on the muscle tissue layer. This experiment was conducted to control the change in DR spectra in the transition from adipose to muscle tissue without any changes of the tissue. Thirty spectra were obtained per experimental condition using the flat-tipped probe.

The third experiment measured the DR spectra of the transition from muscle tissue to adipose tissue layers, including electrosurgical cut top layers. Again, the transition between the adipose tissue and muscle tissue was simulated by removing the sowbelly tissue layers one by one after measuring DR spectra using model 3. However, in this experiment, the top layer was cut each time before measuring the DR spectra. After cutting, the top layer was removed and the cutting was performed at the same spot on the remaining layers. This experiment was less controlled compared to experiment two, however, it corresponds more with reality as it includes electrosurgical cutting. Thirty spectra were obtained per experimental condition using the flat-tipped probe.

In the fourth experiment, the DR spectra were acquired when cutting with the photonic electrosurgical knife with the fibers integrated into the blade (see Fig. 2). This experiment was conducted with the photonic electrosurgical knife connected to a stage that allowed micrometer displacements. The photonic electrosurgical knife was placed perpendicular to the tissue [see Fig. 6(a)] with the initial position slightly into the adipose tissue layer [see Fig. 1(e), model 2]. During $\sim 1 \mathrm{~s}$, the knife was advanced $250 \mu \mathrm{m}$ further into the tissue while cutting at $60 \mathrm{~W}$. After the cutting, five spectra were recorded. This process was repeated until two adipose-muscle transitions were recorded $(\sim 14 \mathrm{~mm})$. The spectra were analyzed to determine the fatto-water fraction.

\subsection{Processing}

In the diffusion theory model developed by Farrell et al. ${ }^{43}$, a DR spectrum is considered the nonlinear product of the absorption and scattering of all the components (chromophores) within the measured spectrum. The FD is an input parameter for the model. In order to match the analytical diffusion equation with the 


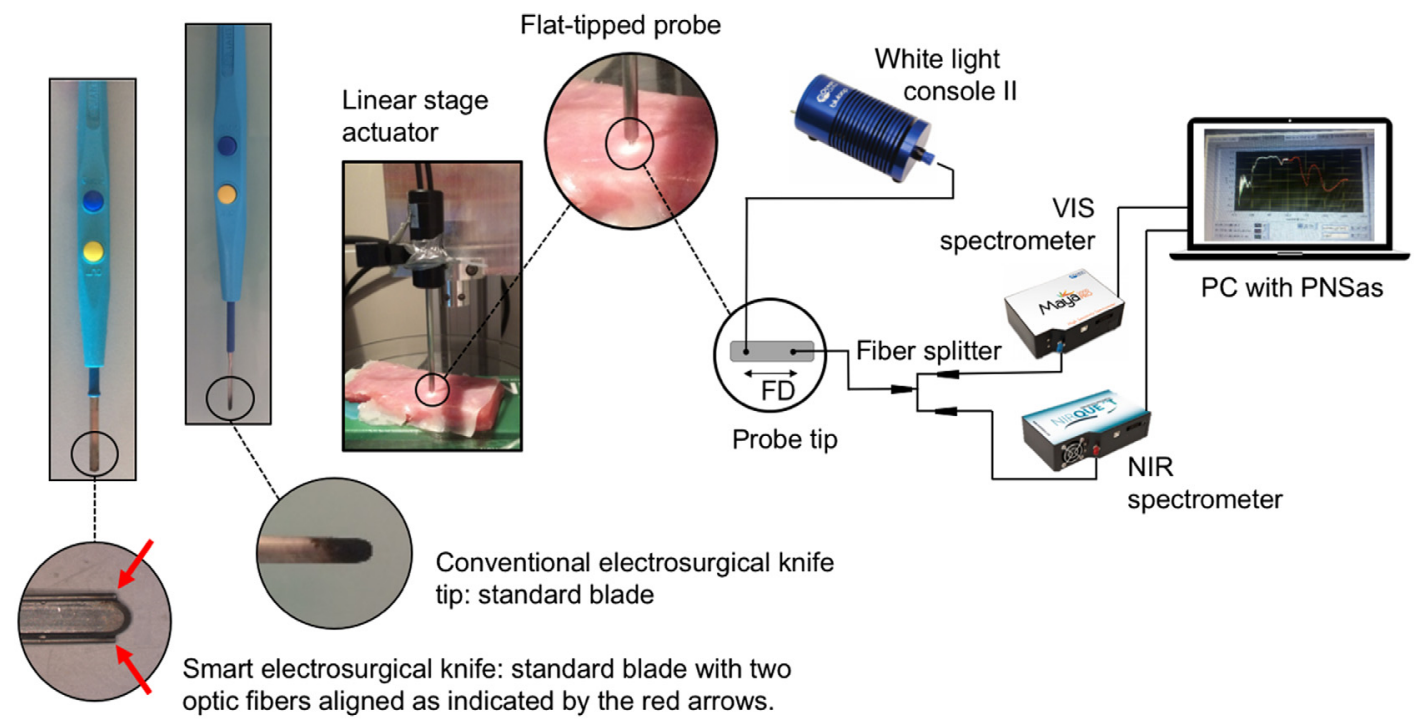

Fig. 2 Overview of the tools used for coagulation and DR measurements.

measured spectrum, an initial guess for the chromophore concentrations (using a priori knowledge of the absorption coefficient of each chromophore) and scattering is made and iteratively updated until the sum of squared error is minimized. A MATLAB-based fitting algorithm was used to obtain the water and fat concentration and scattering parameters (modeled by a Rayleigh and Mie scattering contribution), as described in detail in Ref. 25. Absorption data of tissue chromophores including hemoglobin, oxyhemoglobin, water, fat, and methemoglobin were used as input for the fit sequence. For each parameter, the confidence interval was calculated from the covariance matrix to assess the reliability. ${ }^{25}$ Although the model is derived for a monolayer semi-infinite medium, Monte Carlo calculations showed that the model describes the trends in double-layered tissue configurations well. Apart from model-based extraction of the absorption and scattering coefficient as function of the wavelength, a spectral shape comparison of normal and coagulated tissues was made by normalizing the spectra so that the area under the curve is equal to 1 .

\subsection{Statistics}

Boxplots are used to show the median and interquartile range (IQR) of the data in Figs. 3 and 5, presenting the incision depths after cutting Dsi and coagulation Dsc, the coagulation depth Dxc and the fat-water ratio at a certain distance in the tissue, respectively.

Normality of the data was checked using a one-sample Kolmogorov-Smirnov test, followed by a Mann-Whitney U test to establish whether or not the samples are significantly different. P-values smaller than 0.05 are considered significant.
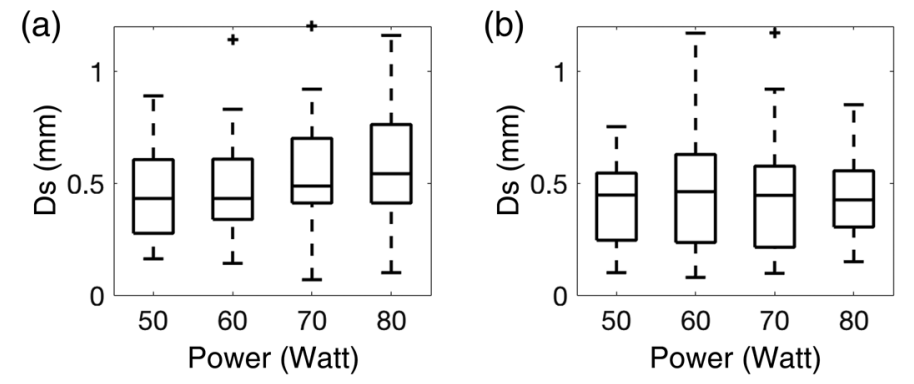

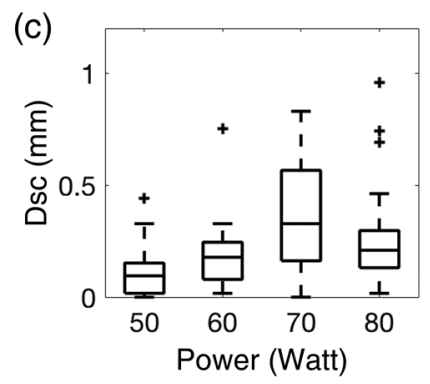

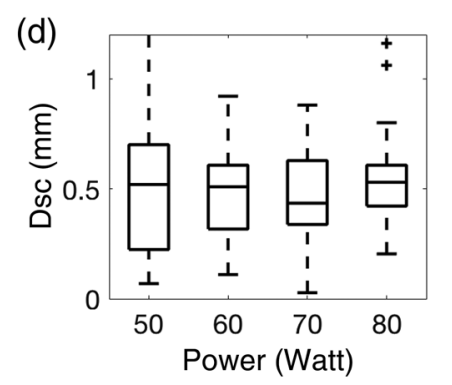

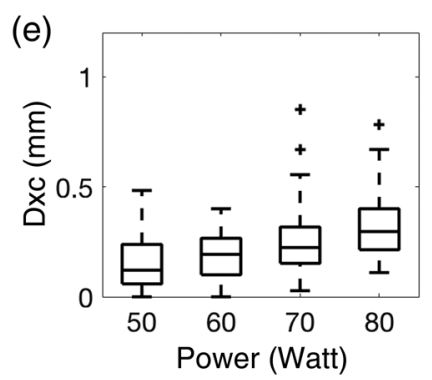

Fig. 3 Boxplots of the incision depth after (a) cutting in muscle Dsi, (b) cutting in adipose tissue Dsi, (c) coagulating muscle Dsc, (d) coagulating adipose tissue Dsc, and (e) boxplot of the coagulation depth after coagulating muscle Dxc. 


\section{Results}

\subsection{Incision Depth for Cutting and Coagulating Muscle and Adipose Tissue}

Figure 3 shows the incision depth and coagulation depth after cutting and coagulating porcine tissue (muscle and adipose) with an electrosurgical knife using various power settings (50 to $80 \mathrm{~W}$ ) for cutting and coagulation. All combinations are performed 10 times. Per sample, 3 measurements were made concluding into 30 measurements per boxplot. The incision depth when cutting [Figs. 3(a) and 3(b)] is comparable for muscle and adipose tissue with a median value of $\sim 0.5 \mathrm{~mm}$. The incision depth is lower for coagulating than for cutting muscle tissue, whereas for adipose tissue the values are comparable. The coagulation depth when coagulating in muscle tissue increases when the power is increased [see Fig. 3(e)]. When cutting muscle tissue, no coagulation zone was found/measured. The median incision depth in adipose tissue appears the same for all power settings for both cutting and coagulation. The coagulation zone is invisible for both cutting and coagulation of adipose tissue.

\subsection{Spectra for Various Power Settings}

Figure 4 shows the normalized spectra of normal muscle and adipose tissue as well as spectra obtained after coagulation with various power settings. Cutting at various powers leads to distinct spectral shape changes in muscle tissue, whereas for adipose tissue only minor changes are observed. During cutting of muscle, the overall spectral shape changes, the blood peaks in the 500- to 600-nm region are less distinct, and the slope of the spectrum for 700 to $900 \mathrm{~nm}$ decreases. The changes for adipose tissue are smaller; a slight decrease in both the peaks in the 500- to 600-nm region and the 1400 - to $1600-\mathrm{nm}$ region, and a small increase between the 800 - and 1100-nm shoulder.

Comparing the changes in DR spectra for muscle and adipose tissue for cutting, the changes are similar before $600 \mathrm{~nm}$, but after $600 \mathrm{~nm}$ the changes in spectra for muscle tissue after cutting are larger.

In Table 1, the fitting results for the parameters related to the NIR part of the spectrum [i.e., reduced scattering coefficient $\mu_{s}^{\prime}$ at $800 \mathrm{~nm}$ and the fat/(fat + water) ratio] for muscle and adipose tissue are listed. For muscle, the scattering increases with
Table 1 Fitting results of the reduced scattering coefficient and the fat/(fat + water) ratio from the measured muscle and adipose tissue spectra at different power settings.

\begin{tabular}{lccccc} 
& \multicolumn{2}{c}{ Muscle tissue } & & \multicolumn{2}{c}{ Fat tissue } \\
\cline { 2 - 3 } \cline { 6 - 6 } $\begin{array}{l}\text { Power } \\
\text { setting (W) }\end{array}$ & $\begin{array}{c}\mu_{s}^{\prime}(800 \mathrm{~nm}) \\
\left(\mathrm{cm}^{-1}\right)\end{array}$ & $\begin{array}{c}\text { Fat/(fat }+ \\
\text { water) }(\%)\end{array}$ & $\begin{array}{c}\mu_{s}^{\prime}(800 \mathrm{~nm}) \\
\left(\mathrm{cm}^{-1}\right)\end{array}$ & $\begin{array}{c}\text { Fat/(fat }+ \\
\text { water) }(\%)\end{array}$ \\
\hline 0 & 4.7 & 18.5 & & 7.0 & 93.1 \\
50 & 6.3 & 25.9 & & 7.7 & 82.4 \\
60 & 7.7 & 24.6 & & 8.5 & 83.7 \\
70 & 9.6 & 26.6 & & 8.9 & 83.0 \\
80 & 10.5 & 33.8 & & 9.2 & 82.0 \\
\hline
\end{tabular}

increasing coagulation power while only a moderate increase is observed for fat. The amount of fat as a fraction of the total fat and water content show a small change when power increases from 0 to $50 \mathrm{~W}$ and remains the same when the power is further increased.

\subsection{Spectra Change at the Transition Between Muscle and Adipose (Sowbelly and Pure) Tissue}

Figure 5(a) shows the spectra obtained at $0 \mathrm{~mm}$ (muscle tissue), $0.4,0.8,1.2,1.6,2$, and $2.4 \mathrm{~mm}$ (sowbelly tissue) with the flattip probe. The transition between the spectra progresses gradually as can be seen in the NIR region, especially between 1200 and $1400 \mathrm{~nm}$. The fat-water ratio is significantly different for all distances in Fig. 5(b) except for the pair 2.0 and $2.4 \mathrm{~mm}$. At $0 \mathrm{~mm}$, the ratio has a median of $10 \%$ (IQR $6 \%$ to $14 \%$ ), which ends at $2.4 \mathrm{~mm}$ with a median of $92 \%$ (IQR $91 \%$ to $93 \%$ ). At $1.6 \mathrm{~mm}$, the transition is saturated.

Figure 5(c) shows the spectra obtained using adipose tissue layers upon muscle tissue. The adipose tissue layers are "purely" cut with the electrosurgical knife operated at $80 \mathrm{~W}$. Again, the spectra show a smooth transition between muscle tissue $(0 \mathrm{~mm})$ and adipose tissue $(2 \mathrm{~mm})$. The fat-water ratio in Fig. 5(d) shows the transition in fat-water ratio obtained for this
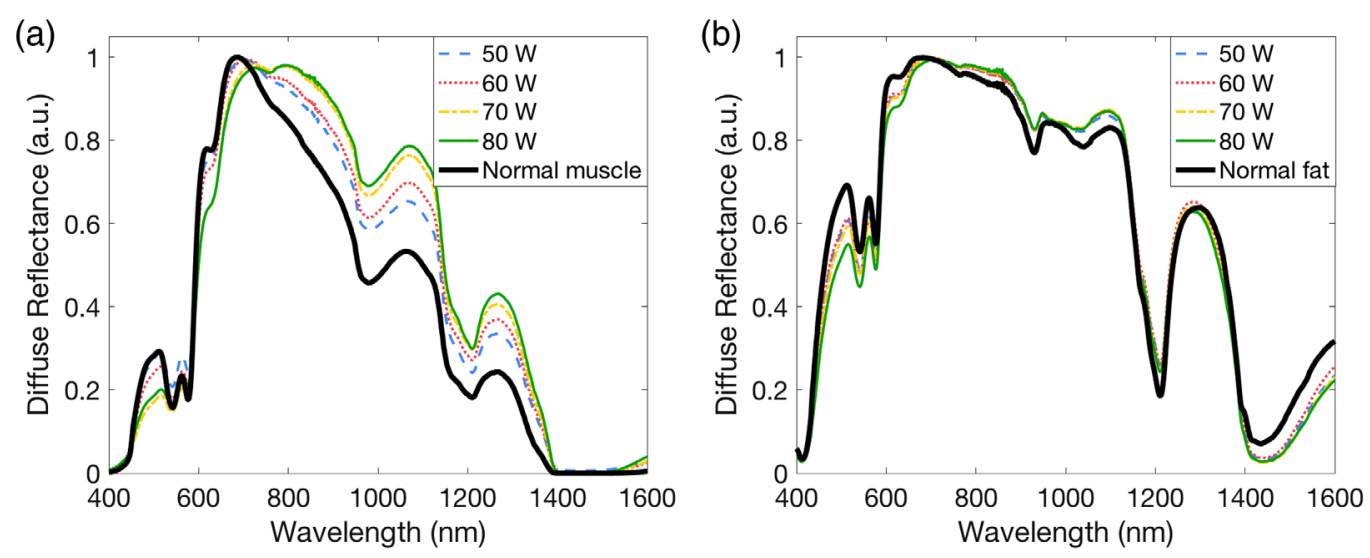

Fig. 4 Spectra for cuts at various power settings at FD $=6.2 \mathrm{~mm}$ for (a) muscle tissue and (b) adipose tissue. For these experiments, 2-cm layers of muscle and/or $2 \mathrm{~cm}$ of adipose porcine tissue were used (model 2). 

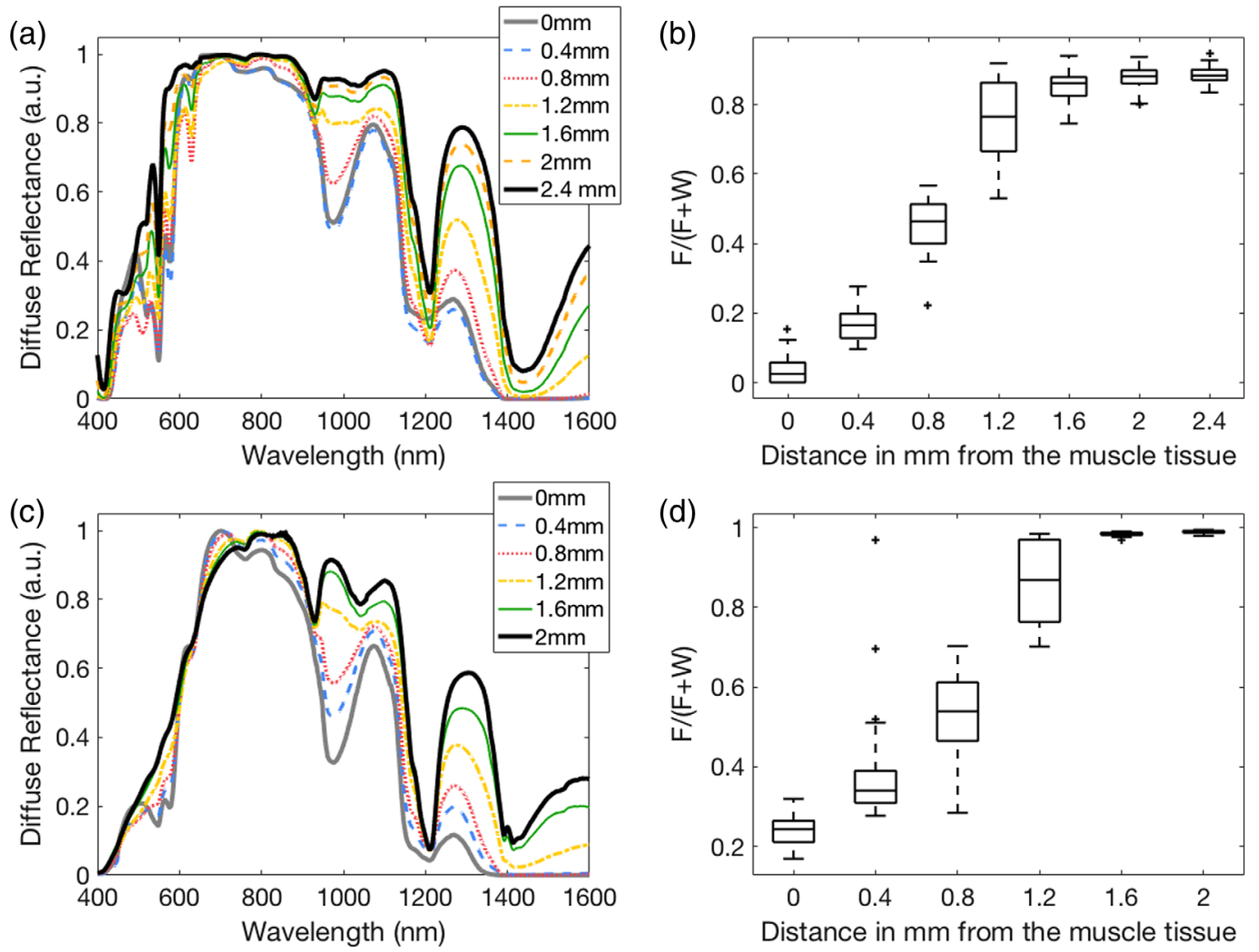

Fig. 5 (a) Spectra and (b) fat/(water + fat) ratio of muscle $(0 \mathrm{~mm})$ under $0.4-\mathrm{mm}$ layers of noncut adipose tissue $(0.4$ to $2.4 \mathrm{~mm}$ ) and (c) spectra and (d) fat/(water + fat) ratio of muscle $(0 \mathrm{~mm})$ under $0.4-\mathrm{mm}$ layers of cut adipose tissue $(0.4$ to $2 \mathrm{~mm}$ ). For these experiments, a 2-cm layer of muscle tissue and 0.4-mm layers of adipose porcine tissue were used (model 3).

experiment. All ratios are significantly different. At $0 \mathrm{~mm}$, the ratio has a median of $10 \%$ (IQR $6 \%$ to $14 \%$ ), which ends at $2 \mathrm{~mm}$ with a median of $100 \%$ (IQR $100 \%$ to $100 \%$ ) At $1.6 \mathrm{~mm}$, the transition is saturated.

Figure 6 shows the results when performing the optical measurements during cutting at $60 \mathrm{~W}$ into porcine belly tissue, where the optical fibers are integrated into the electrosurgical knife. The knife was advanced through alternating layers of adipose and muscle tissue [see Fig. 6(a), using model 2]. The fat fraction measured [see Fig. 6(b)] shows a transition zone of $\sim 1 \mathrm{~mm}$ when the photonic electrosurgical knife was advanced from one layer to the other. Furthermore, the fat fraction values determined from the measurements in adipose tissue show less variation than in muscle tissue. Figure 6(c) shows typical measured spectra from 500 to $1600 \mathrm{~nm}$ at various depth locations with the same acquisition times. For wavelength lower than $500 \mathrm{~nm}$, the noise in the spectra increases due to the decrease in detector efficiency. The spectra measured in muscle tissue show a lower intensity than those in adipose tissue. Furthermore, the typical blood absorption dips of (oxy)hemoglobin for wavelengths between 500 and $600 \mathrm{~nm}$ are not observed in Fig. 5. Except for the wavelength region 900 to $1100 \mathrm{~nm}$ for the spectrum at 1-mm-depth distance, the fitted results are in good agreement with the measured spectra.

\section{Discussion}

In this study, the possibility of using DRS as a guiding tool in the tip of an electrosurgical instrument was examined through tissue experiments focusing on the size and shape of the coagulation zone and spectral measurements of layered tissues. The measurements showed that coagulation significantly alters the optical properties of the visible part of the spectrum. The fat/water ratio determined from the NIR part of the spectrum was hardly affected by coagulation. A non-negligible coagulation zone was only observed in muscle with typical size of $0.2 \mathrm{~mm}$ when using the electrosurgical knife in coagulation mode. Measurements on the transition from adipose-to-muscle with or without a coagulation layer showed the same trends in the fat/water ratio. The photonic electrosurgical knife was able to detect the transition between these layers based on the fat/water ratio when cutting through alternating layers of adipose and muscle porcine tissue.

\subsection{Incision Depth for Cutting and Coagulating Muscle and Adipose Tissue}

The incision depth increases with an increase in power from 50 to $80 \mathrm{~W}$ when cutting and coagulating muscle tissue (Fig. 3). However, this is only true for muscle tissue; as for adipose tissue, the incision depth does not change when increasing the power setting. It is expected that the incision depth will achieve an equilibrium when using higher powers in muscle tissue. The variance in incision depth seems to increase when increasing the power in both muscle and adipose tissue [Figs. 3(a) and 3(c)]. The accuracy of depth measurements is limited due to irregularity and nonsmoothness of the tissue. In addition, measurements of the incision depth provided limited sample rates. However, for the purpose of obtaining 

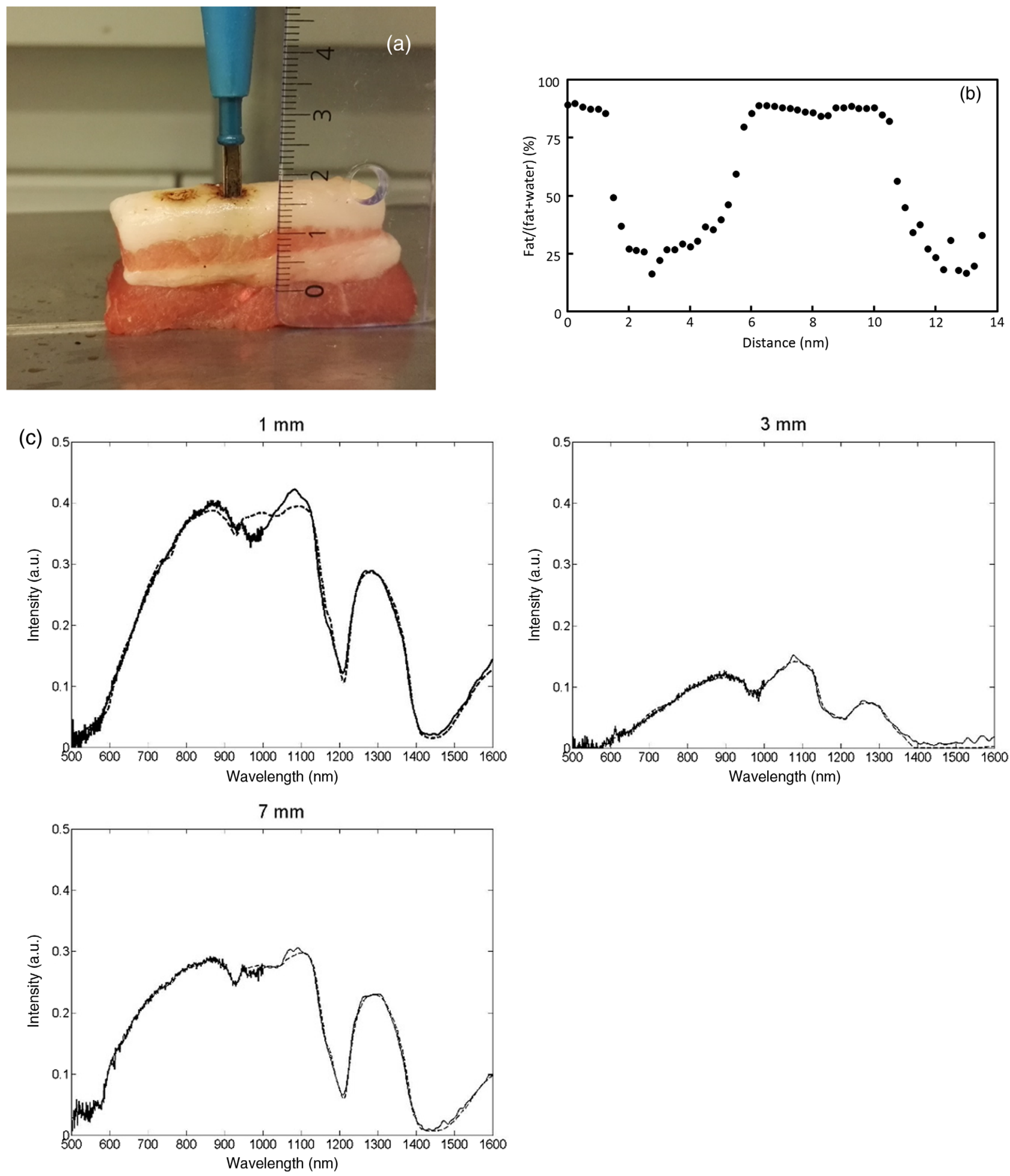

Fig. 6 (a) Photograph of the electrosurgical knife with tissue sensing when cutting into swine tissue, (b) the measured fat fraction as function of the cutting depth, and (c) the measured spectra (solid line) and fitted result (dashed line) for several depth distance.

a range likely encountered in surgery, these results are deemed sufficient. When coagulating in muscle, the incision depth is smaller than for cutting at the expense of creating a coagulation zone [Fig. 3(e)]. For adipose tissue, no coagulation zone is created. Apparently, the energy deposited in the adipose tissue results mainly in melting and solidifying of the adipose tissue.

\subsection{Spectra for Coagulated Tissue with Various Power Settings}

Coagulated tissue produced a relatively stronger signal in the NIR region, with increased peaks around 1095 and $1280 \mathrm{~nm}$ and a less steep slope in the VIS region. The increase is called relative because of the normalization of setting the area under 
the curve to 1 , which negates the effects of overall spectral intensity increase or decrease and only allows comparison of relative changes in spectral shapes. Overall spectral intensity depends on many factors including pressure on the tissue, sensitivity of sensors, and ambient light. Overall intensity is a useful parameter for within-sample measurements such as in Fig. 6, while spectral shapes are better suited to compare measurements taken from different samples. Table 1 shows that for muscle the scattering increases due to coagulation, with reduced water content after coagulation. This is in accordance with the protein denaturation and evaporation events that are known to occur during tissue heating. ${ }^{44-46}$ Spectral changes for coagulated adipose tissue are limited to small intensity changes while the overall shape is maintained. This is explained by the fact that adipose tissue melts when it is heated, causing little to no changes with respect to the presence of chromophores in the tissue. The protein content of adipose tissue is much lower than for muscle resulting in only moderate changes in the scattering coefficient. Coagulation is known to affect the blood composition. For instance, hemoglobin changes partially into methemoglobin and other blood derivatives accounting for the changes in the 450- to 700-nm wavelength range of the spectrum. ${ }^{47}$ For both muscle and adipose tissue, the fat-to-water ratio is only moderately affected by the coagulation. These observations are in line with the results of the study on the effects of cauterization on the optical properties on breast specimens by Bydlon et al. ${ }^{48}$.

Due to the large fiber distances used, the coagulated spectra in Fig. 4 are probably the result of a mixed signal: coagulated tissue from the top layer and normal tissue from the bottom layer. This agrees with the situation that is encountered in actual surgery and these spectra allow us to predict and understand trends from coagulation.

\subsection{Spectra Change at the Transition Between Muscle and Adipose (Sowbelly and Pure) Tissue}

The spectra in Fig. 5(a) show a gradual shape transition from adipose to muscle tissue, which is similar to previous studies performed on layered tissue. ${ }^{49,50}$ The layer of adipose tissue on top of the muscle tissue consists of separate layers of 0.4$\mathrm{mm}$ thick slices of adipose tissue. This stacking of separate layers caused increased scattering due to the small air inclusions between the layers compared to one solid adipose tissue layer. As a result, a rather small sensing depth of $1.6 \mathrm{~mm}$ is found compared to results found by Hennessy et al. ${ }^{51}$. In addition, increased scattering lowers the peaks around $500 \mathrm{~nm}$.

The same shape transition is observed in Fig. 5(c), where the top layer has been cut. These layers can show more effects of coagulation as the top layer was cut and the layers underneath were not renewed for new experiments so they were coagulated multiple times. Again, the increased scattering caused by stacking of separate adipose tissue layers causes a small sensing depth of $1.6 \mathrm{~mm}$ and decreases peaks around $500 \mathrm{~nm}$. Other small changes can be present due to variations in the tissue.

In Fig. 6, the tissue measurements were performed in alternating layers of adipose and muscle tissue with the optical fibers directly integrated in the electrosurgical knife. Based on the fat/ water ratio, the transitions between the alternating adipose and muscle layers are well visible. Although the fat/water ratio remains a stable parameter during this cutting process, the intensity of the spectra shows significant variation. Inspection of the fiber ends showed the presence of contaminations of the cutting process. Although this had minor effect on the fat/water ratio and the fitting of the spectra, this remains a point for further attention. Especially in situations where protein and blood rich tissue are cut, debris may contaminate the fiber ends.

These measurements were performed on ex vivo tissue, which is not perfused with blood as is the case in surgery. For in vivo measurements, we expect a different signal in the 400- to 600-nm blood region both due to the difference in amount of blood and blood oxygenation as well as the formation of debris on the electrosurgical knife due to cutting in blood rich tissue. Furthermore, increased amount of methemoglobin and derivative formation after coagulation is likely to occur in vivo. This is expected to mainly affect the shape of the visible part of the spectrum and the total intensity with less impact on the fat-water ratio as can be seen in Fig. 6(c). Human adipose tissue contains beta-carotene, a chromophore that absorbs mostly in the lower VIS region. Also, in vivo coagulation creates a transition of hemoglobin to methemoglobin, creating an extra spectral feature in the VIS region as shown by Spliethoff et al. ${ }^{47}$ The presence of beta-carotene and (met)hemoglobin will likely influence the spectral shape in the VIS region but not the fat/ water ratio extraction. Furthermore, the measurements were performed using perfectly flat geometries. In the tissue cuts, it was observed that the coagulated layer is rarely completely flat, even as a result of very steady knife-handling. Therefore, it is expected that geometry resulting from surgical electrocoagulation will be more irregular. As a result, the electrosurgical knife might not always be in optimal contact with the tissue and the influence of this partial contact combined with nonflat layers should be further investigated.

de Boer et al. ${ }^{30}$ showed that breast tumor boundaries can be detected based on the fat-to-water ratio extracted from the measured DR spectra. During breast tumor resection, a surgeon will typically remove adipose tissue past the boundary of the tumor, in an effort to resect any microscopic tumor extensions. Such "negative margins" have been shown to reduce the possibility of reoccurrence of the tumor. The measurements performed in this paper where obtained while cutting in adipose tissue toward muscle tissue. This fat-to-muscle model is a good representation for the fat-to-breast tumor transition in the wavelength reason 1000 to $1600 \mathrm{~nm}$ region but is less representative for the difference in the visible part of the spectrum. The measurements showed that the photonic electrosurgical knife is able to detect these adipose/muscle boundaries even in the presence of cut tissue. Since the fat-to-water ratio is hardly affected by the cutting this demonstrates that the photonic electrosurgical knife has the potential to perform real-time breast tumor detection during the cutting process without the need of a separate probe as studied in de Boer et al. ${ }^{30}$. This study was focused on breast tumors, simulated using adipose and muscle tissue. The benefits of using DRS may also improve other oncological procedures such as colorectal tumors surrounded by adipose tissue and prevent iatrogenic damage to nerves and blood vessels in bariatric surgery, thyroidectomy, and prostatectomy.

\subsection{Future Developments of a Photonic Electrosurgical Knife}

After performing the experiments described in this paper, some practical remarks on the future design of the photonic electrosurgical knife can be made. The optic fibers were positioned right next to the electrosurgical blade, so that the fiber tips were always in contact with the tissue at the same time as 
the blade. This resulted in small amounts of coagulated or charred tissue sticking to the fiber, which leads to undesired alterations in the DR signal and possible damage to the fiber tips. The amount of coagulation seems to depend on the tissue type being cut, the setting and power setting of the knife and the number of times the knife was used. To prevent the fibers from being damaged, the fiber tips should be a small distance away from the blade tip during cutting and coagulation. During measurements the fibers should be in contact with the tissue, directed in such a way that they register the tissue in the cutting direction. This could also prevent the fibers being damaged during cleaning of the blades for which normally an abrasive pad is used in the operating room.

The median cutting depth for adipose tissue found in this paper is $0.45 \mathrm{~mm}$ for a power setting of 50 to $80 \mathrm{~W}$. However, the variance increased when higher power settings were used [Figs. 2(a) and 2(c)]. Therefore, it is important to keep the power setting in mind for the use of the photonic electrosurgical knife. The tissue layer being coagulated while cutting is negligible. For coagulation of the tissue, the median of the coagulated layer is 0.2 to $0.3 \mathrm{~mm}$ for 50 to $80 \mathrm{~W}$. The effect of coagulation on the tissue is small, and therefore, the underlying tissue can still be detected. However, the presence of this small layer and its effect on the DR signal has to be taken into account when setting up an algorithm for tumor margin detection.

\section{Conclusion}

In this study, the influence of cutting and coagulation on the DR spectra of muscle and adipose tissue was examined. The coagulation zone for cutting and coagulating in muscle tissue is small and practically nonexistent in adipose tissue. Using the fat/water ratio from DR spectra of cut layered tissues is an effective method for tissue transition detection. First experiments showed that the fat-to-water ratio is hardly affected by the presence of cut and coagulated tissue. Therefore, an electrosurgical knife equipped with optical fibers for obtaining DR spectra is a feasible and promising method for detecting tissue boundaries determined by changes in fat content. In conclusion, the photonic electrosurgical knife has the potential to provide realtime feedback about the presence of breast tumor margins during cutting, helping surgeons to establish negative margins and improve patient outcome.

\section{Disclosures}

None of the other authors have any financial relationship with Philips Research or conflict of interests.

\section{Acknowledgments}

This study was supported by Philips Research, Eindhoven, Netherlands. The authors who are affiliated with Philips Research only have financial interests in the subject matter, materials, and equipment, in the sense that they are an employee of Philips.

\section{References}

1. N. Howlader et al., "SEER cancer statistics review, 1975-2014," National Cancer Institute, https://seer.cancer.gov/csr/1975_2014/ (based on November 2016 SEER data submission, posted to the SEER web site, April 2017).

2. M. Mohs et al., "Hand-held spectroscopic device for in vivo and intraoperative tumor detection: contrast enhancement, detection sensitivity, and tissue penetration," Anal. Chem. 82(21), 9058-9065 (2010).
3. K. D. Miller et al., "Cancer treatment and survivorship statistics, 2016," CA. Cancer J. Clin. 66(4), 271-289 (2016).

4. M. De Grand and J. V. Frangioni, "An operational near-infrared fluorescence imaging system prototype for large animal surgery," Technol. Cancer Res. Treat. 2(6), 553-562 (2003).

5. M. Sampo et al., "Impact of the smallest surgical margin on local control in soft tissue sarcoma," Br. J. Surg. 95(2), 237-243 (2008).

6. E. Sadot et al., "Resection margin and survival in 2368 patients undergoing hepatic resection for metastatic colorectal cancer: surgical technique or biologic surrogate?" Ann. Surg. 262(3), 476-485 (2015).

7. M. S. Moran et al., "Society of surgical oncology-American Society for Radiation Oncology consensus guideline on margins for breastconserving surgery with whole-breast irradiation in stages I and II invasive breast cancer," Int. J. Radiat. Oncol. Biol. Phys. 88(3), 553-564 (2014).

8. M. Pilewskie and M. Morrow, "Extent and role of margin control for DCIS managed by breast-conserving surgery," in Ductal Carcinoma In Situ and Microinvasive/Borderline Breast Cancer, L. Newman and J. Bensenhaver, Eds., pp. 67-83, Springer, New York (2015).

9. M. D. Mc Kee et al., "The prognostic significance of margin width for extremity and trunk sarcoma," J. Surg. Oncol. 85(2), 68-76 (2004).

10. P. I. Tartter et al., "Lumpectomy margins, reexcision, and local recurrence of breast cancer," Am. J. Surg. 179, 81-85 (2000).

11. A. Kobbermann et al., "Impact of routine cavity shave margins on breast cancer re-excision rates," Ann. Surg. Oncol. 18, 1349-1355 (2011).

12. M. van der Noordaa et al., "The use of radioactive iodine- 125 seed localization in patients with non-palpable breast cancer: a comparison with the radioguided occult lesion localization with $99 \mathrm{~m}$ technetium," Eur. J. Surg. Oncol. 41(4), 553-558 (2015).

13. N. Krekel et al., "Excessive resections in breast-conserving surgery: a retrospective multicentre study," Breast J. 17(6), 602-609 (2011).

14. S. Luini et al., "Comparison of radioguided excision with wire localization of occult breast lesions," Br. J. Surg. 86(4), 522-525 (1999).

15. M. Thill, K. Baumann, and J. Barinoff, "Intraoperative assessment of margins in breast conservative surgery-still in use?" J. Surg. Oncol. 110, 15-20 (2014).

16. T. Vo-Dinh, Biomedical Photonics Handbook: Biomedical Diagnostics, Vol. 2, CRC press, Boca Raton, Florida (2014).

17. J. Q. Brown et al., "Optical assessment of tumor resection margins in the breast," IEEE J. Sel. Top. Quantum Electron. 16, 530-544 (2010).

18. A. S. Haka et al., "In vivo margin assessment during partial mastectomy breast surgery using Raman spectroscopy," Cancer Res. 66(6), 33173322 (2006).

19. J. Balog et al., "Intraoperative tissue identification using rapid evaporative ionization mass spectrometry," Sci. Transl. Med. 5(194), 194ra93 (2013).

20. P. B. van Driel et al., "Characterization and evaluation of the Artemis camera for fluorescence-guided cancer surgery," Mol. Imaging Biol. 17(3), 413-423 (2015).

21. T. M. Allweis et al., "A prospective, randomized, controlled, multicenter study of a real-time, intraoperative probe for positive margin detection in breast-conserving surgery," Am. J. Surg. 196(4), 483-489 (2008).

22. J. Alexander et al., "A novel methodology for in vivo endoscopic phenotyping of colorectal cancer based on real-time analysis of the mucosal lipidome: a prospective observational study of the iKnife," Surg. Endosc. 31(3), 1361-1370 (2017).

23. T. M. Bydlon et al., "Chromophore based analyses of steady-state diffuse reflectance spectroscopy: current status and perspectives for clinical adoption," J. Biophotonics 8, 9-24 (2015).

24. D. J. Evers et al., "Optical spectroscopy: current advances and future applications in cancer diagnostics and therapy," Future Oncol. 8(3), 307-320 (2012).

25. R. Nachabé et al., "Diagnosis of breast cancer using diffuse optical spectroscopy from 500 to $1600 \mathrm{~nm}$ : comparison of classification methods," J. Biomed. Opt. 16(8), 087010 (2011).

26. D. J. Evers et al., "Diffuse reflectance spectroscopy: towards clinical application in breast cancer," Breast Cancer Res. Treat. 137(1), 155-165 (2013).

27. E. Tanis et al., "In vivo tumor identification of colorectal liver metastases with diffuse reflectance and fluorescence spectroscopy," Lasers Surg. Med. 48(9), 820-827 (2016). 
28. D. Evers et al., "Optical sensing for tumor detection in the liver," Eur. J. Surg. Oncol. 39(1), 68-75 (2013).

29. L. De Boer et al., "Margin assessment in breast cancer lumpectomy specimens with infrared diffuse reflectance spectroscopy (DRS)," Ann. Oncol. 25(Suppl 4), iv105 (2014).

30. L. L. de Boer et al., "Fat/water ratios measured with diffuse reflectance spectroscopy to detect breast tumor boundaries," Breast Cancer Res. Treat. 152(3), 509-518 (2015).

31. N. van de Berg et al., "Energetic soft-tissue treatment technologies: an overview of procedural fundamentals and safety factors," Surg. Endosc. 27(9), 3085-3099 (2013).

32. M. G. Munro, "Fundamentals of electrosurgery part 1: principles of radiofrequency energy for surgery," in The SAGES Manual on the Fundamental Use of Surgical Energy (FUSE), L. Feldman, P. Fuchsuber, and B. Jones, Eds., Springer, New York (2012).

33. S. L. Jacques, "Role of tissue optics and pulse duration on tissue effects during high-power laser irradiation," Appl. Opt. 32(13), 2447-2454 (1993).

34. K. Roggan et al., "The optical properties of biological tissue in the near infrared wavelength range-review and measurements," in LaserInduced Interstitial Therapy, G. Muller and A. Roggan, Eds., CRC Press, Berlin, Germany (1995).

35. P. Yaroslavsky et al., "Optical properties of selected native and coagulated human brain tissues in vitro in the visible and near infrared spectral range," Phys. Med. Biol. 47(12), 2059-2073 (2002).

36. G. J. Derbyshire, D. K. Bogen, and M. Unger, "Thermally induced optical property changes in myocardium at $1.06 \mu \mathrm{m}$," Lasers Surg. Med. 10(1), 28-34 (1990).

37. J.-P. Ritz et al., "Optical properties of native and coagulated porcine liver tissue between 400 and $2400 \mathrm{~nm}$," Lasers Surg. Med. 29(3), 205-212 (2001).

38. L. S. Feldman, P. R. Fuchshuber, and D. B. Jones, Eds., The SAGES Manual on the Fundamental Use of Surgical Energy (FUSE) Springer, New York (2012).

39. C. W. Van Way, "Electrosurgery 101," Curr. Surg. 57(2), 172-177 (2000).

40. T. Alkatout et al., "Principles and safety measures of electrosurgery in laparoscopy," J. Soc. Laparoendosc. Surg. 16(1), 130-139 (2012).

41. P. Taheri et al., "Electrosurgery: Part II. Technology, applications, and safety of electrosurgical devices," J. Am. Acad. Dermatol. 70(4), 607.e1-607.e12 (2014).

42. R. D. Tucker, "Laparoscopic electrosurgical injuries: survey results and their implications," Surg. Laparosc. Endosc. Percutaneous Tech. 5(4), 311-317 (1995).

43. T. J. Farrell, M. S. Patterson, and B. Wilson, "A diffusion theory model of spatially resolved, steady-state diffuse reflectance for the noninvasive determination of tissue optical properties in vivo," Med. Phys. 19(4), 879-888 (1992).

44. G. Sankaranarayanan et al., "Common uses and cited complications of energy in surgery," Surg. Endosc. 27(9), 3056-3072 (2013).
45. T. L. Smith and J. M. Smith, "Electrosurgery in otolaryngologyhead and neck surgery: principles, advances, and complications," Laryngoscope 111(5), 769-780 (2001).

46. P. Taheri et al., "Electrosurgery: Part I. Basics and principles," J. Am. Acad. Dermatol. 70(4), 591.e1-591.e14 (2014).

47. W. Spliethoff et al., "Monitoring of tumor radio frequency ablation using derivative spectroscopy," J. Biomed. Opt. 19(9), 097004 (2014).

48. T. M. Bydlon et al., "Advancing optical imaging for breast margin assessment: an analysis of excisional time, cautery, and patent blue dye on underlying sources of contrast," PLoS One 7(12), e51418 (2012).

49. H. Arimoto, M. Egawa, and Y. Yamada, "Depth profile of diffuse reflectance near-infrared spectroscopy for measurement of water content in skin," Skin Res. Technol. 11(1), 27-35 (2005).

50. M. Sharma et al., "Verification of a two-layer inverse Monte Carlo absorption model using multiple source-detector separation diffuse reflectance spectroscopy," Biomed. Opt. Express 5(1), 40-53 (2014).

51. R. Hennessy et al., "Effect of probe geometry and optical properties on the sampling depth for diffuse reflectance spectroscopy," J. Biomed. Opt. 19(10), 107002 (2014).

Maartje W. Adank obtained her MSc degree in biomedical engineering with a speciality in Medical Instruments and Medical Safety at Delft University of Technology (DUT) in 2017. After completing she started working at Brainlab as an Application Consultant for Image Guided Systems.

Julie C. Fleischer is a researcher in the field of global surgery at the Department of Biomechanical Engineering, DUT and cofounder of Opt Medical. She obtained her MSc degree in Biomechanical Engineering at DUT in 2015. Her current work focuses on low-cost equipment for difficult airways (DUT) and novel contraceptive solutions for low- and middle-income countries (Opt Medical).

Jenny Dankelman is professor in Minimally Invasive Surgery and Interventional Techniques at the Delft University of Technology. She was awarded the Antoni van Leeuwenhoek chair in 2001 and she became Medical Delta professor in 2013. Her research group cooperates with several hospitals such as Leiden UMC, where she holds a part time professorship position, Erasmus MC Rotterdam and the AMC Amsterdam. Her interests and research projects are in the fields of designing novel medical instruments, haptics, training and simulation systems, and patient safety, with the focus on minimally invasive techniques.

Benno H. W. Hendriks is a researcher at Philips Research Eindhoven the Netherlands and professor in Optics for Minimally Invasive Instruments at the Department of Biomechanical Engineering, DUT. He obtained his MSc degree in theoretical physics at RU Utrecht in 1985 and $\mathrm{PhD}$ on quantum optics at RU Utrecht in 1989. His current work focuses on light-tissue interactions for biomedical application, image guided therapy solutions and minimally invasive devices for medical applications. 